\title{
0386 FALL INJURIES IN INDIA: A REVIEW
}

S Malhotra*, R Ivers Correspondence: Indian Institute of Public Health, Public Health Foundation of India, B-3/84 A MIG DDA Flats Keshav Puram, Lawrence Road, New Delhi, 110035, India

10.1136/ip.2010.029215.386

A review study was carried out on fall injuries in Indian context to synthesise evidence, using electronic and manual search, in English language with key [MeSH] words: accidental falls and India. The review aimed to capture information on burden, risk factors, fall prevention interventions carried out in India. The studies available for the review were predominantly cross sectional hospital based studies. In a National report in 2007, fall injuries was responsible for $3.3 \%$ of deaths and less than $1 \%$ of injuries, highlighting an underestimated figure and problems in official data. A comprehensive community based study in rural Andhra Pradesh reported fall injuries to be second most common (20.1\%) and was commonest non-fatal injury (38.8\%) reported among all injuries. As per Bengaluru Injury Surveillance Programme, there were $9 \%$ deaths and $10 \%$ hospitalisations in a ratio of 1:25 for fatal and non-fatal injuries. Data from other hospital based studies also point out towards commonality of fall injuries among children and elderly especially females. Limited small scale studies have been done to associate falls and resulting injuries, disabilities, psychological stress due to brain; maxillofacial trauma and fractures. Chronic coexistent illnesses, use of drugs, poor gait and joint problems among elderly, alcohol in adults and poor housing situation in children are risk factors associated with falls. Limited evidence exists in regard to interventions and its impact in the population. There is an urgent need to carry out larger community based epidemiological studies to fill the information gaps. 